\title{
Occulting Focal Plane Masks for Terrestrial Planet Finder Coronagraph: Design, Fabrication, Simulations and Test Results
}

\author{
Kunjithapatham Balasubramanian ${ }^{1}$, Daniel J. Hoppe ${ }^{1}$, Peter G. \\ Halverson $^{1}$, Daniel W. Wilson ${ }^{1}$, Pierre M. Echternach ${ }^{1}$, Fang Shi ${ }^{1}$, \\ Andrew E. Lowman ${ }^{1}$, Albert F. Niessner ${ }^{1}$, John T. Trauger ${ }^{1}$, and \\ Stuart B. Shaklan ${ }^{1}$ \\ ${ }^{1}$ Jet Propulsion Laboratory, California Institute of Technology 4800 Oak Grove Drive, \\ Mail Stop 301-451, Pasadena, CA 91109-8099, USA \\ email: kbala@jpl.nasa.gov
}

\begin{abstract}
Occulting focal plane masks for the Terrestrial Planet Finder Coronagraph (TPF-C) could be designed with continuous gray scale profile of the occulting pattern such as $1-\operatorname{sinc}^{2}$ on a suitable material or with micron-scale binary transparent and opaque structures of metallic pattern on glass. We have designed, fabricated and tested both kinds of masks. The fundamental characteristics of such masks and initial test results from the High Contrast Imaging Test bed (HCIT) at JPL are presented.
\end{abstract}

Keywords. instrumentation: high angular resolution, instrumentation: adaptive optics, instrumentation: miscellaneous.

\section{Introduction}

Occulting focal plane masks operate in conjunction with a Lyot stop to suppress the starlight in a coronagraph (Kuchner \& Spergel 2003). In the limit of Fourier Optics, these masks can easily be designed to reject the starlight in the regions of interest to below $10^{-10}(-100 \mathrm{~dB})$, the nominal Terrestrial Planet Finder (TPF) requirement (Kuchner \& Traub 2002). Band-limited gray scale and binary masks have been designed, fabricated and tested to meet such a requirement. In this paper, we describe the design, fabrication and test results.

\section{HEBS Mask Design and Fabrication}

Analog gray scale masks based on a special high energy electron beam sensitive (HEBS) glass can be designed to work as focal plane occulting masks to achieve the required suppression of star light through iterative development of the material, design, and fabrication. Wilson et al. (2003) and Halverson et al. (2005) have reported on the fabrication and characteristics of HEBS masks for TPF applications. Early experimental results with HEBS masks were also reported by Trauger et al. (2004). Band limited focal plane masks with $1-\operatorname{sinc}^{2}$ transmission profiles discussed by Kuchner \& Traub (2002) could be designed and fabricated on HEBS glass with electron beam exposure techniques. Such masks designed to operate in the high contrast imaging test bed at JPL were fabricated at the Micro Devices Lab (MDL) in JPL; transmission image and cross section profile of one such mask fabricated and tested are shown in Figure 2. Several masks have been 


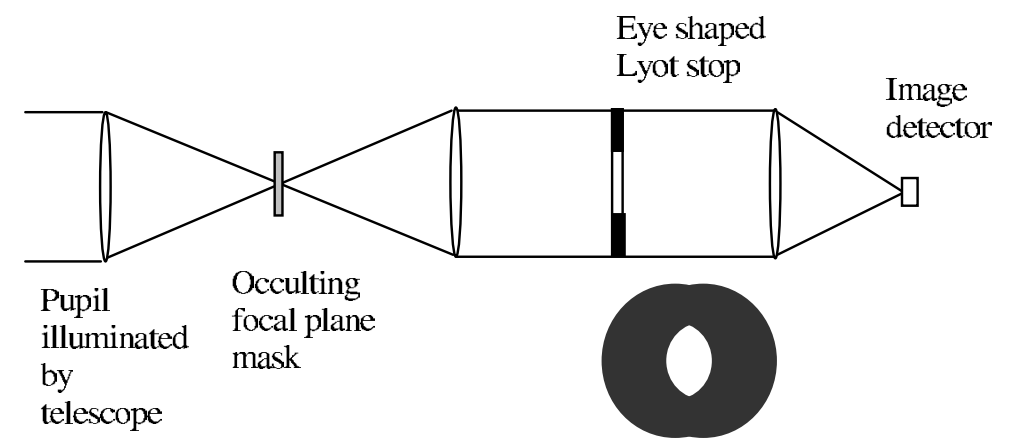

Figure 1. Conceptual drawing of a coronagraph with focal plane mask and Lyot stop.
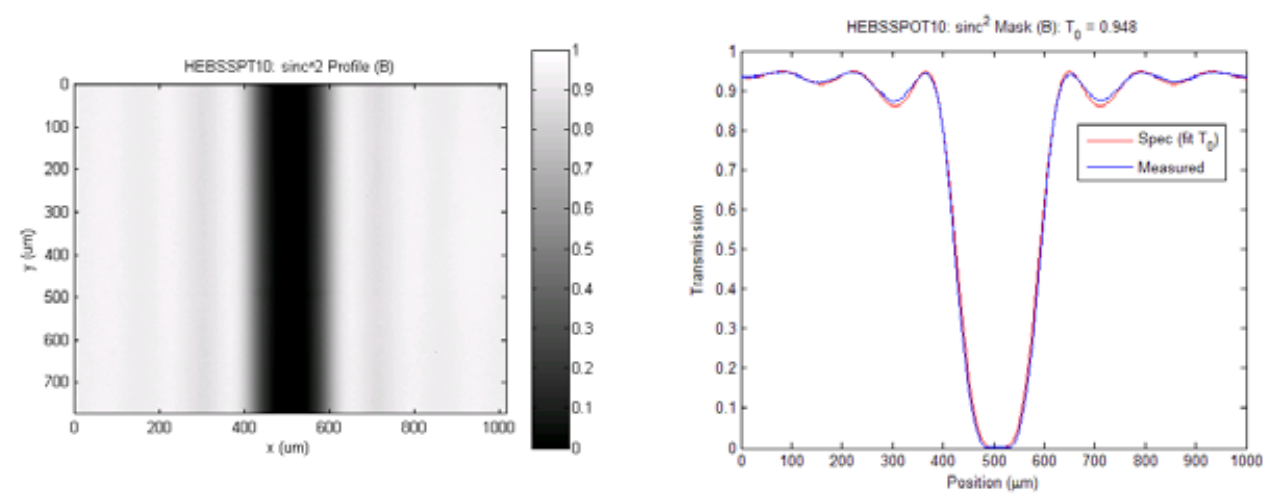

Figure 2. Typical transmission image (left panel) and profile (right panel) of $1-\operatorname{sinc}^{2}$ HEBS mask profile for $785 \mathrm{~nm}$.

fabricated with profiles given by:

$$
T(x)=T_{0}\left[1-\left(\frac{\sin (\pi x / w)}{\pi x / w}\right)^{2}\right]^{2} .
$$

The width parameter $w$ for one of the masks is $141.9 \mu \mathrm{m}, T(x)=0.5 T_{0}$ at $x=81.3 \mu \mathrm{m}$. $T(x)$ was set to 1.0 for all $|x|>1.20 \mathrm{~mm} ; T_{0}=0.90$. A similar profile with $w=157.7 \mu \mathrm{m}$ and $T(x)=0.5 T_{0}$ at $x=90.3 \mu \mathrm{m}$ has also been fabricated. These profiles were designed for $F=f / D=28.55$ and $\lambda=785 \mathrm{~nm}$.

\section{High Energy Beam sensitive Glass (HEBS) Mask Fabrication}

The HEBS glass as produced by Canyon Materials Inc. $\dagger$ consists of a $10 \mu \mathrm{m}$ thick high energy electron beam sensitive layer on both sides of a 90 mil $(2.29 \mathrm{~mm})$ thick glass plate. The sensitive layer gets darkened to various levels of optical density when exposed to high energy electron beam irradiation at chosen doses. Specific transmission profiles can be obtained on such a glass when the electron beam is scanned on the glass to dosage levels preprogrammed to produce the darkness of the required mask transmission profile (Wilson et al. 2003; Halverson et al. 2005). Our masks were fabricated with a high precision electron beam lithography system (JEOL 9300FSZ Electron Beam Lithography

$\dagger$ Canyon Materials, Inc. San Diego, CA http://canyonmaterials.com 


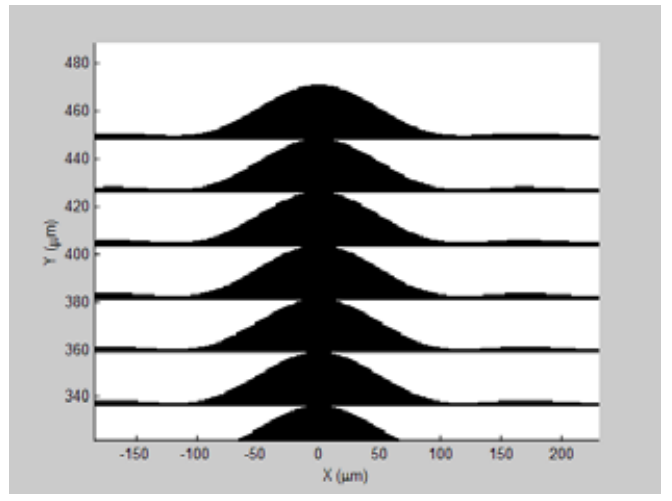

Figure 3. Detailed view of binary $1-\operatorname{sinc}^{2}$ mask pattern.

at Micro Devices Lab) with $100 \mathrm{kV}$ beam at $1 \mathrm{nA}$ current. Calibration runs guide the choice of e-beam exposure parameters such as current, exposure time, scan step etc to achieve a desired optical density at a specific location on a mask. Effects of convolution of the e-beam spot size, scan steps and proximity effects are taken in account in calibrating the exposure and scan parameters.

\section{Binary Mask Design and Fabrication}

In the case of binary masks, an analog band-limited function such as $\sin ^{2}$ or $1-$ $\operatorname{sinc}^{2}$ is sampled and a series of apertures is created that mimics the throughput of the analog mask. Sampled and continuous $\sin ^{2}$ and $\operatorname{sinc}^{2}$ type masks have been designed and fabricated. The mask is referred to as binary since it consists only of perfectly blocking metal and totally transmitting empty apertures $(0 / 1)$. The binary masks are chosen to be periodic in the non-search direction $(y)$, with a period of $F \lambda$, where $F=f / D$ is the focal length/diameter ratio of the telescope and $\lambda$ is the operating wavelength. To match the performance of the above HEBS mask designs, binary masks were designed for the same $F$ and $\lambda$. Figure 3 shows the design of a binary mask made to mimic the $1-\operatorname{sinc}^{2}$ transmission profile of the HEBS mask. The binary mask patterns are generated by e-beam lithography and lift off process with nominally $150 \mathrm{~nm}$ thick aluminum film coated by evaporation on fused silica glass.

\section{High Contrast Imaging Testbed (HCIT)}

The HEBS and binary masks described above were tested for performance in the HCIT at JPL. The test bed set up has been described by Trauger et al. (2004) and in the proceedings of this conference (IAUC200). A recent addition to the test bed optics is a calcite polarizer before the final image detector. This polarizer separates the two orthogonally polarized images into slightly offset locations at the final image plane. The deformable mirror (DM) can be optimized to minimize the speckles in either polarization independently, thus estimating the best contrast achievable in one polarization while allowing the other to float. While the HEBS gray scale mask does not exhibit polarization sensitivity, the binary mask is inherently sensitive to polarization. 


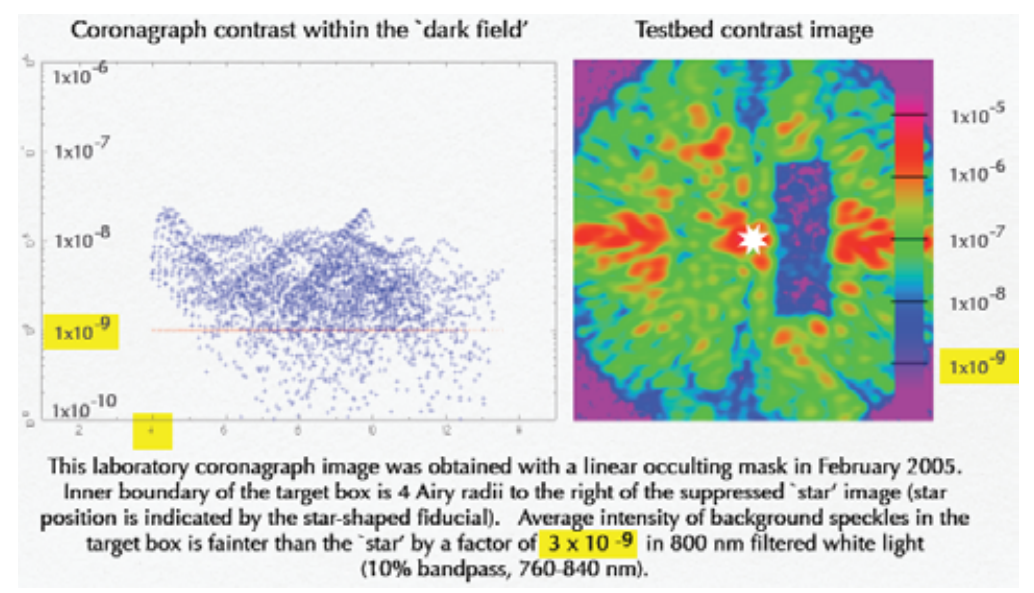

Figure 4. HEBS mask HCIT contrast vs. Airy radius in the target box of 4 to $10 \lambda / D$ in $x$ and -10 to $+10 \lambda / D$ in $y$.

\section{Experimental Results}

Contrast images were obtained with a HEBS $1-$ sinc $^{2}$ linear occulting mask with $785 \mathrm{~nm}$ laser source in the HCIT. An average contrast of $9 \times 10^{-10}$ was achieved with a stability of about $5 \times 10^{-11}$ per hour. Similar experiments with the same mask with filtered white light source with $10 \%$ band pass centered at $800 \mathrm{~nm}$ achieved average contrast of $3 \times 10^{-9}$ in the target box. Figure 4 shows the contrast image and plot for this result. Latest experiments have yielded results in the $10^{-10}$ scale as presented by Trauger et $a l$. at this conference. The binary $1-\operatorname{sinc}^{2}$ mask was tested in the HCIT under similar conditions as with the HEBS mask. With incident $785 \mathrm{~nm}$ unpolarized laser light and split into orthogonal polarizations after the mask and before the final image, a contrast of $4 \times 10^{-9}$ was obtained in the target box for a chosen polarization that was nulled with the DM while the other polarization was left floating (Figure 5). The experiment in the HCIT collected two images (Figure 5, left) corresponding to the two orthogonal polarization states after the calcite crystal. Theoretical simulations with Fresnel diffraction models compare closely with the experimental results; observed differences between theory and experiment particularly with respect to the polarization behavior of binary masks may be explained as due to model assumptions and experimental conditions which are being further analyzed.

\section{Acknowledgements}

This work was performed at the Jet Propulsion Laboratory, California Institute of Technology, under a contract with the National Aeronautics and Space Administration. Reference herein to any specific commercial product, process or service by trade name, trademark, manufacturer, or otherwise, does not constitute or imply its endorsement by the United States Government or the Jet Propulsion Laboratory, California Institute of Technology. The authors thank Brian Kern of the Caltech Jet Propulsion Laboratory for his help with reviewing and editing this paper in $\mathrm{LAT}_{\mathrm{E}} \mathrm{X}$ format to suit publication.

\section{References}

Kuchner, M. J. \& Spergel, D. N. 2003, "Notch Filter Masks: Practical Image Masks for PlanetFinding Coronagraphs," ApJ 594, 617 

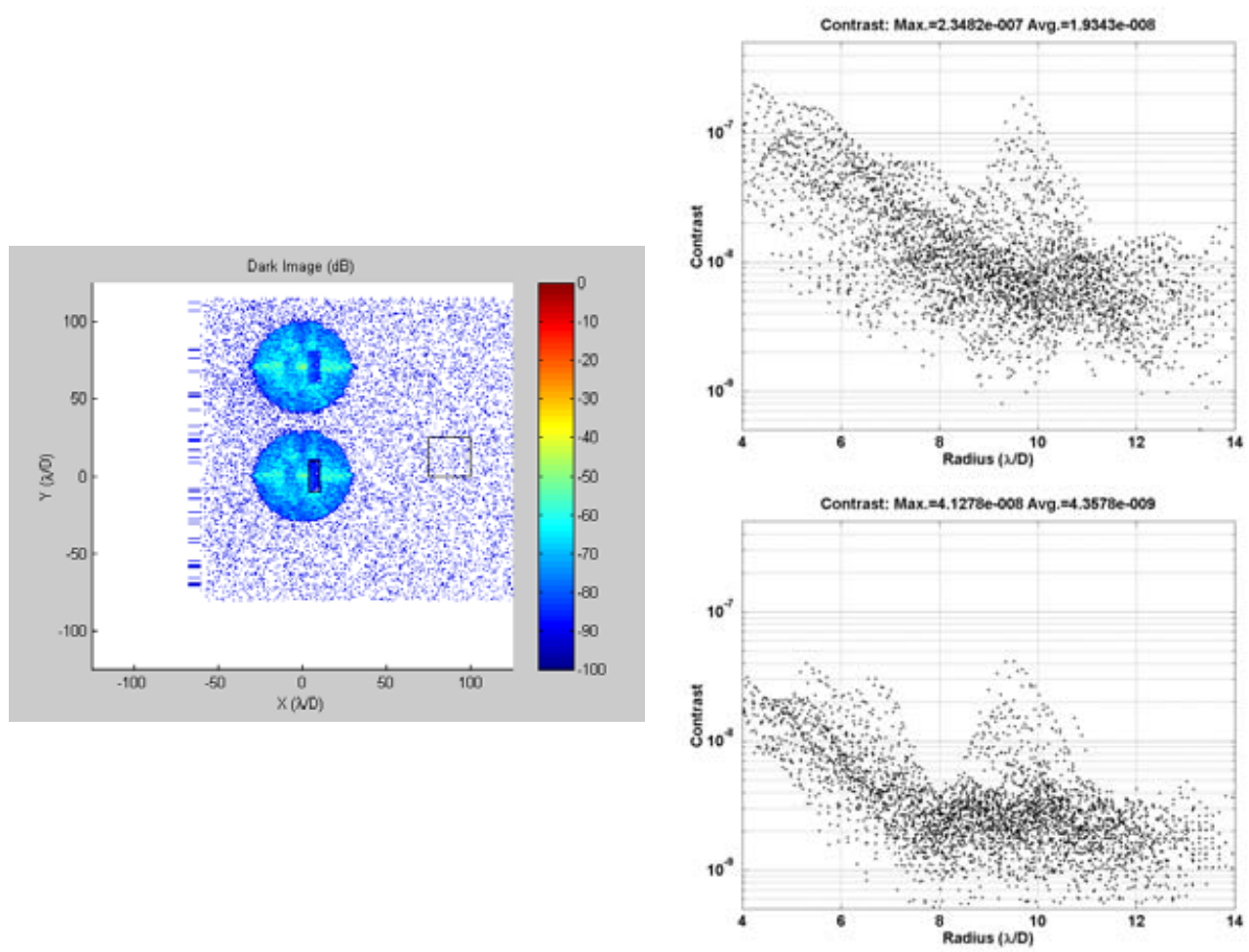

Figure 5. LEFT: Final experimental images showing two polarizations of binary mask. The experimentally measured average contrast in the polarization being nulled (bottom of image) is $4.3 \times 10^{-9}$, whereas the average contrast in the orthogonal field not nulled (top of image) by DM is $1.9 \times 10^{-8}$. TOP RIGHT: Contrast plot of polarization not being nulled by DM settings. BOTTOM RIGHT: Contrast plot of polarization being nulled by DM.

Kuchner, M. J. \& Traub, W. 2002, "A coronagraph with a band-limited mask for finding terrestrial planets," ApJ 570, 900

Wilson, D. W. et al. 2003, "Eclipse apodization: realization of occulting spots and Lyot masks," Proc. SPIE 4860, 361

Halverson, P. G. et al. 2005, "Measurement of phase and optical density of TPF coronagraph occulting mask materials," Proc. SPIE 5905

Trauger, J. T. et al. 2004, "Coronagraph contrast demonstrations with the High Contrast Imaging Testbed," Proc. SPIE 5487, 133. Also latest results were presented in this IAU colloquium.

Wilson, D. W. et al. 2005, "Electron-beam lithography for micro- and nano-optical applications," Proc. SPIE 5720, 68 




All photographs: Laurent Thareau [1.thareau@free.fr]. 